\title{
Molecular expression of multiple Nav1.5 splice variants in the frontal lobe of the human brain
}

\author{
JUN WANG, SHAO-WU OU, ZHI-YONG ZHANG, BO QIU and YUN-JIE WANG \\ Department of Neurosurgery, The First Hospital of China Medical University, Shenyang, Liaoning 110001, P.R. China
}

Received March 19, 2017; Accepted November 22, 2017

DOI: $10.3892 /$ ijmm.2017.3286

\begin{abstract}
Voltage-gated sodium channels serve an essential role in the initiation and propagation of action potentials for central neurons. Previous studies have demonstrated that two novel variants of Nav1.5, designated Nav1.5e and Nav1.5f, were expressed in the human brain cortex. To date, nine distinct sodium channel isoforms of Nav1.5 have been identified. In the present study, the expression of Nav1.5 splice variants in the frontal lobe of the human brain cortex was systematically investigated. The results demonstrated that wild Nav1.5 and its splice variants, Nav1.5c and Nav1.5e, were expressed in the frontal lobe of the human brain cortex. Nav1.5a, Nav1.5b and Nav1.5d splice variants were not detected. However, the expression level of different Nav1.5 variants was revealed to vary. The expression ratio of wild Nav1.5 vs. Nav1.5c and Nav1.5e was approximately 5:1 and 1:5, respectively. Immunochemistry results revealed that Nav1.5 immunoreactivity was predominantly in neuronal cell bodies and processes, including axons and dendrites, whereas little immunoreactivity was detected in the glial components. These results revealed that a minimum of four Nav1.5 splice variants are expressed in the frontal lobe of the human brain cortex. This indicates that the previously reported tetrodotoxin-resistant sodium current was a compound product of different Nav1.5 variants. The present study revealed that Nav1.5 channels have a more abundant expression in the human brain than previously considered. It also provided further insight into the complexity and functional significance of Nav1.5 channels in human brain neurons.
\end{abstract}

\section{Introduction}

Voltage-gated sodium channels (VGSCs) serve a key role in the initiation and propagation of action potentials in neurons (1-4). These channels consist of a major pore-forming $\alpha$-subunit (Nav1; $\sim 260 \mathrm{kDa}$ ) and 1-2 accessory $\beta$-subunits (4).

Correspondence to: Professor Yun-Jie Wang, Department of Neurosurgery, The First Hospital of China Medical University, 155 Nanjing North Street, Shenyang, Liaoning 110001, P.R. China

E-mail: woaishoushu@hotmail.com

Key words: voltage-gated sodium channel, Nav1.5, splice variants, brain cortex
The $\alpha$-subunit is the core of the sodium channel and contains the elements essential for ion conduction and voltage-dependent gating (4-6). To the best of our knowledge, at present 10 distinct sodium channel isoforms (Nav1.1-1.9 and NavX) and four small $\beta$-subunits $(\beta 1-4 ; 30.4-45 \mathrm{kDa})$ have been detected and cloned from various mammalian tissues $(5,6)$. Tetrodotoxin (TTX) is a specific inhibitor against sodium channels; VGSCs are classified into TTX-sensitive (Nav1.1-1.4, Nav1.6 and Nav1.7) and TTX-resistant or insensitive (Nav1.5, Nav1.8 and Nav1.9) types (5).

The intrinsic electrophysiological properties of human neurons are complex (1). Therefore, it is important to clarify the distinct isoforms of sodium channels expressed in the human brain in order to explore the contribution of specific VGSCs to the excitability of central neurons. Among the 10 sodium channel isoforms, Nav1.1-1.3 and Nav1.6 were the first cloned from the brain and functionally analyzed, as they were originally designated as brain sodium channel types I, II, III and IV (7-9). However, previous studies indicated that Nav1.5 mRNA, protein and its TTX-resistant sodium currents may also be detected in the mammalian brain and associated cell lines (10-22). In a previous study, we cloned the full length of Nav1.5 cDNA and demonstrated that two novel variants of Nav1.5, designated Nav1.5e and Nav1.5f, were expressed in the human brain cortex $(23,24)$. To the best of our knowledge, at present, nine Nav1.5 splice variants, including Nav1.5a-f and truncated variants Nav1.5 E28B-D have been identified, four of which (Nav1.5a and Nav1.5c-e) are able to generate functional channels in heterologous expression systems (25). However, the electrophysiological properties vary among different Nav1.5 splice variants $(26,27)$. As the different expression patterns of Nav1.5 splice variants may affect the total Nav1.5 sodium current density and kinetics in human brain neurons, the present study investigated whether additional Nav1.5 splice variants were expressed in the human brain cortex. Therefore, the present study systematically investigated the expression of Nav1.5 splice variants, including the functional isoforms in the frontal lobe of the human brain cortex. In addition, the expression and distribution of total Nav1.5 protein in the neurons and glial cells of the gray and white matter within the human brain cortex was detected.

\section{Materials and methods}

Materials. The present study conformed to the principles outlined in the declaration of Helsinki, and was approved by 
the Ethics Committee of China Medical University (Shenyang, China). Five human brain samples were collected from the First Hospital and the Department of Anatomy of China Medical University (Shenyang, China) between January 2013 and December 2015. The samples were collected from 3 males and 2 females, with an age range of 43-78 years. Three samples (frontal lobe cortexes) were obtained at autopsy from adults without a history of neurologic diseases at the Department of Anatomy of China Medical University. All autopsies were performed within $12 \mathrm{~h}$ of mortality. Two human brain samples were collected blindly from discarded tissues from surgeries to treat basilar meningiomas at the First Hospital of China Medical University. All samples were collected with prior understanding and written informed consent from the patients or their relatives.

RNA isolation and reverse transcription-polymerase chain reaction $(R T-P C R)$. Total RNA was extracted from human brain cortexes using an RNA Extraction kit (Takara Bio, Inc., Otsu, Japan) according to the manufacturer's protocol. First-strand cDNA was synthesized using an RT-PCR kit (New England BioLabs, Inc., Ipswich, MA, USA) with oligo-(dt) and random primers. The $\mathrm{RT}$ reaction conditions consisted of $30^{\circ} \mathrm{C}$ for $5 \mathrm{~min}$ followed by $42^{\circ} \mathrm{C}$ for $25 \mathrm{~min}, 99^{\circ} \mathrm{C}$ for $5 \mathrm{~min}$ and $5^{\circ} \mathrm{C}$ for $5 \mathrm{~min}$. For the detection and isolation of Nav1.5 splice variants from the human brain cortex, primer pairs P1-P6 (Table I) were used to amplify different fragments of the full-length Nav1.5 cDNA by the PCR method. The PCR primers were designed from the full sequences of the SCN5A gene cloned from the human brain cortex (accession number, EF629346) and the PCR was performed separately, using a One-Taq One-Step RT-PCR kit (New England Biolabs, Inc.), according to the specific reannealing temperatures of different primers. The PCR reaction conditions were as follows: $95^{\circ} \mathrm{C}$ for $5 \mathrm{~min}$ followed by 36 cycles of $95^{\circ} \mathrm{C}$ for $30 \mathrm{sec}, 60-66^{\circ} \mathrm{C}$ for $30 \mathrm{sec}, 72^{\circ} \mathrm{C}$ for $30 \mathrm{sec}$ and final elongation at $72^{\circ} \mathrm{C}$ for $5 \mathrm{~min}$.

DNA sequencing. The PCR products were separated by electrophoresis on a $2 \%$ agarose gel. The different fragments of the expected size were extracted by using a gel extraction kit (Qiagen, Inc., Valencia, CA, USA) and subsequently sequenced directly using a 3730xl DNA Analyzer (Applied BioSystems; Thermo Fisher Scientific, Inc., Waltham, MA, USA), according to the manufacturer's protocol.

Denaturing gel electrophoresis and DNA argentation. Denaturing gel electrophoresis and the DNA argentation method was used to detect the relative quantity of alternatively spliced transcripts of Nav1.5. Denaturing gel electrophoresis was performed at room temperature, at $5 \mathrm{~W}$ for $4 \mathrm{~h}$ on a $12 \%$ polyacrylamide (19:1), $7 \mathrm{M}$ urea gel. Subsequently, $2 \% \mathrm{AgNO}_{3}$ was used for the silver staining test of DNA at room temperature.

Restriction enzyme digestion. Restriction enzyme SacI (cat. no. D1078A; Takara Bio, Inc., Otsu, Japan) was used to digest the PCR products to distinguish between different splice variants of Nav1.5. The total reaction system was $30 \mu 1$ [4 $\mu 1$ PCR products, $0.5 \mu \mathrm{l}$ SacI enzyme, $3 \mu \mathrm{l}$ loading buffer (Takara Bio, Inc.); $22.5 \mu \mathrm{l}$ ultrapure water]. Following $1 \mathrm{~h}$ incubation at $38^{\circ} \mathrm{C}$, electrophoresis was performed on $2 \%$ agarose gel to detect the digestion results. The expression ratio of Nav1.5 variants vs. total Nav1.5 was detected from the signal quantification of pre- and post-digestion by autoradiography.

Immunostaining. Human brain cortex specimens were fixed with $4 \%$ paraformaldehyde $\left(4^{\circ} \mathrm{C}\right.$ for $\left.24 \mathrm{~h}\right)$ immediately after collection. The tissues were paraffin-embedded after dehydration using a series of graded ethanol baths (70-100\% ethanol), clearing (the transparency of tissue) using xylene (100\%) and wax infiltration. The streptavidin-peroxidase (SP) immunohistochemical method was applied. Non-immune goat serum (cat. no. ZLI-9022; without dilution; OriGene Technologies, Inc., Beijing, China) was added for blocking followed by incubation at room temperature in a moisture chamber for 30 min. Immunohistochemistry was performed using a Histostain-SP kit (Invitrogen; Thermo Fisher Scientific, Inc.) according to manufacturer's protocol. Sections $(4 \mu \mathrm{m})$ were incubated overnight with rabbit anti-human Nav1.5 antibodies (cat. no. ASC-013; 1:100; Alomone Labs, Jerusalem, Israel) at $4^{\circ} \mathrm{C}$ in a moisture chamber, followed by three washes with PBS, 5 min each. Subsequently, sections were incubated with biotinylated goat anti-rabbit immunoglobulin G secondary antibody (cat. no. 656140; 1:500; Invitrogen; Thermo Fisher Scientific, Inc.) at room temperature for $30 \mathrm{~min}$, followed by three washes with PBS, 5 min each. PBS replaced the primary antibody to serve as a negative control and a slide with known positive Nav1.5 expression in the rat atrial muscle served as a positive control. The immunohistochemical staining results were observed using a light microscope (magnification, x40 and x100; Olympus CX31-LV320; Olympus Corporation, Tokyo, Japan). The expression of brown and yellow staining on the cells was considered to be positive immunoreactions when compared with negative and positive slices.

\section{Results}

Expression of adult and neonatal Navl.5 in the human brain cortex. Primer P1 targeting exon 6 or 6 A of SCN5A was used to detect the expression of adult (wild) and neonatal (Nav1.5e) Nav1.5 in the frontal lobe cortex of human brains. Nav1.5 protein encoded by the SCN5A gene including exon 6 is universally designated as wild or adult Nav1.5, whereas that including exon 6A is universally designated neonatal Nav1.5 or Nav1.5e (28). Electrophoresis revealed that a single clear band with the expected size was visible on the agarose gel (Fig. 1A). Direct DNA sequencing of these PCR products revealed that a single sequence presented in the coding region of exons 5 and 7 , whereas a dual sequence appeared in the coding region of exons 6 and 6A (Fig. 1B). DNA sequence analysis revealed that exon 6 and $6 \mathrm{~A}$ were inclusively expressed, indicating the expression of adult and neonatal Nav1.5 channels in the frontal lobe of the human brain. Exon $6 \mathrm{~A}$ and 6 each contained 92 nucleotides; however, 31 of them were different in various positions (Fig. 1C). The 92 nucleotides encoded 30 amino acid residues. A total of 7 amino acid residues were different between exon 6 and $6 \mathrm{~A}$.

In order to demonstrate the expression quantification of the two splice variants of Nav1.5, the sequence differences between exon 6 and $6 \mathrm{~A}$ were analyzed. Occasionally, a specific 
Table I. Primer sequences used for the isolation of Nav1.5 splice variants.

\begin{tabular}{|c|c|c|c|c|c|}
\hline \multirow[b]{2}{*}{ Primer } & \multicolumn{2}{|c|}{ Primer sequence $\left(5^{\prime}-3^{\prime}\right)$} & \multirow[b]{2}{*}{ Target } & \multirow[b]{2}{*}{ Location $^{\mathrm{a}}$} & \multirow{2}{*}{$\begin{array}{c}\text { Length } \\
\text { (bp) }\end{array}$} \\
\hline & Forward & Reverse & & & \\
\hline P1 & ACCAACTGCGTGTTCATGGCCCA & AGGTCCAGGGATTCCCAGACCA & Exon $6 / 6 \mathrm{~A}$ & $425-916$ & 492 \\
\hline $\mathrm{P} 2$ & ACCAACTGCGTGTTCATGGCCCA & GCAGAAGACTGTGAGGACCA & Exon $6 / 6 \mathrm{~A}$ & $425-778$ & 354 \\
\hline P3 & GTGCCTCCCACCCGCAAGGAAA & TGCTGCCCTCGGAGCAACTGT & Nav1.5a, Nav1.5c & $3054-3420$ & 367 \\
\hline P4 & CTGGGGAACCTGACACTGGTGC & AGATGATGAATGTCTCGAACC & Nav1.5b & $2514-3636$ & 1,123 \\
\hline P5 & CCAAGAAGAGGATGAGGAGA & GAGGCAGTCGCTGACACC & Nav1.5c & $3172-3307$ & $132 / 135$ \\
\hline P6 & TTCAGTGCAGACAACCTCACA & TGTTCTCСТCATCCTCTTCTT & Nav1.5d & 2823-3195 & 373 \\
\hline
\end{tabular}

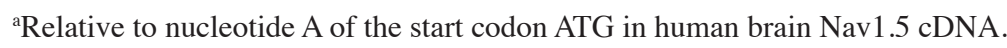
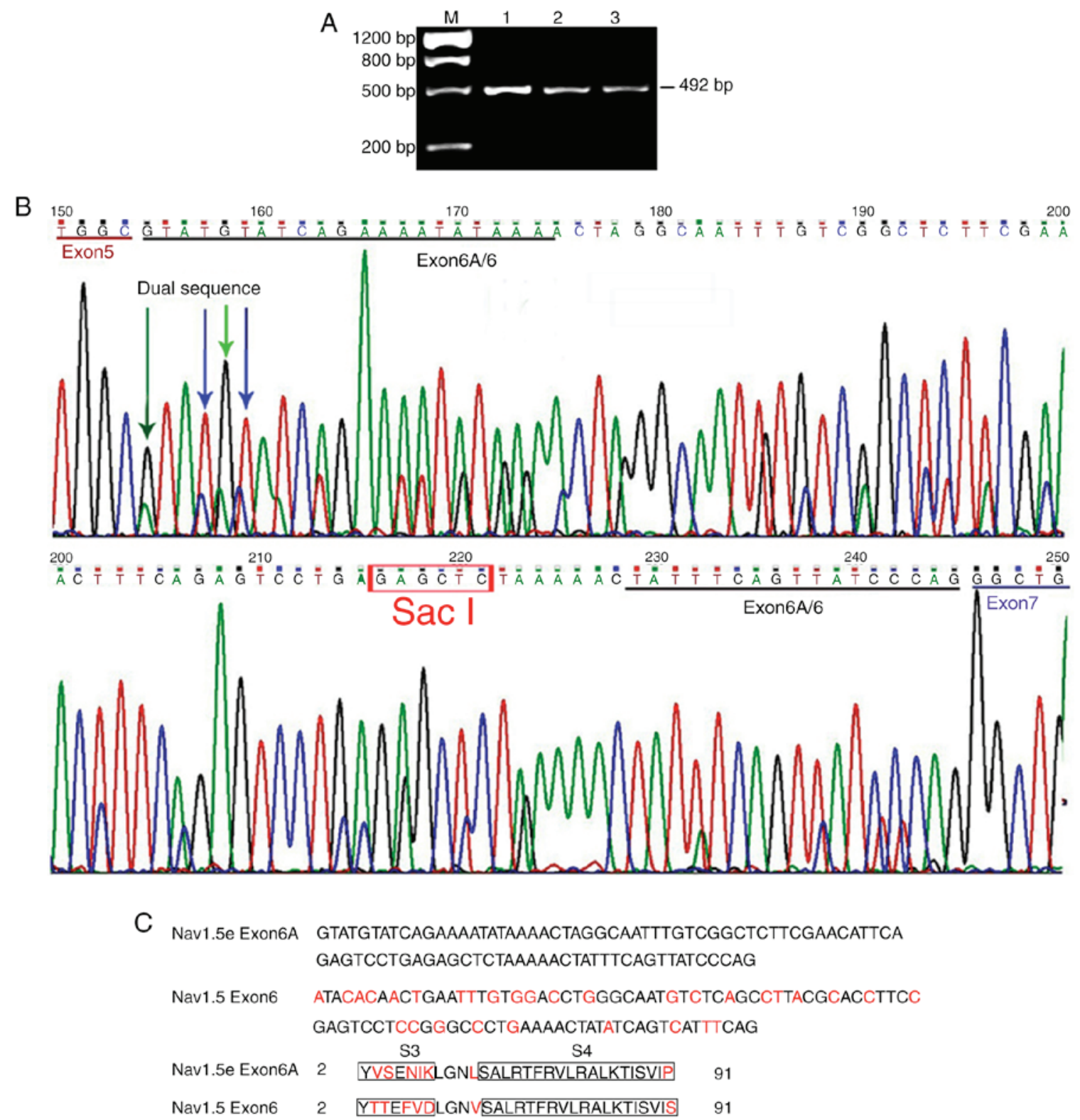

Figure 1. Adult and neonatal Nav1.5 are expressed in the human brain cortex. (A) Electrophoresis on an agarose gel following reverse transcription-polymerase chain reaction with primer P1 revealed a single, clear band of $492 \mathrm{bp}$ in size. (B) Direct DNA sequencing was performed on the purified PCR products. Results revealed that a single sequence was present in the exon 5 or 7 coding region and a dual sequence was observed in the exon 6 coding region. DNA sequence analysis indicated that exon 6 and 6A were expressed in the human brain cortex. (C) Structures of exon 6 and 6 A of SCN5A are depicted. Exon 6 and 6 A contained 92 nucleotides. The red letters indicate the nucleotides $(n=31)$ and amino acid residues $(n=7)$ that differed between the exons. M, marker; lanes 1-3, samples of human brain cortex from the frontal lobe. 
restriction enzyme site of $S a c I$ was identified in exon 6A rather than exon 6 . The restriction enzyme $\mathrm{SacI}$ was subsequently used to digest the PCR products to distinguish between these two variants. The common cDNA from the RT of mRNA and the PCR products generated by primer P1 (which were demonstrated by DNA sequencing to include exon 6 and $6 \mathrm{~A}$ ), were used independently as the templates to perform the usual PCR and the nested PCR. The PCR products (with primers P1 and P2) containing the total Nav1.5 fragments were digested by the restriction enzyme $\mathrm{SacI}$. As revealed in Fig. 2, three bands were observed on the agarose gel following digestion by $\mathrm{SacI}$, which indicated that fragments containing exon $6 \mathrm{~A}$ were digested into two smaller fragments, whereas those containing exon 6 were preserved. Therefore, the relative amounts of digested and undigested PCR products represented the quantification of neonatal and adult Nav1.5 cDNA, respectively. Similar results were observed by using two pairs of primers (P1 and P2) as aforementioned. The expression ratio of neonatal Nav1.5 vs. adult Nav1.5 was $\sim 5: 1$, as calculated from the signal quantification of pre- and post-digestion by autoradiography $(n=5)$ (data of expression ratio not shown).

Expression of Nav1.5a in the human brain cortex. Primer P3 that targeted part of exon 17 and all of exon 18 of Nav1.5 cDNA was used to detect the expression of Nav1.5a splice variant (with the alternative splice of exon 18). PCR products were purified and sequenced directly. The expected fragment size of $367 \mathrm{bp}$ was observed following electrophoresis (Fig. 3). The fragment with alternative splicing of exon 18 (159-bp deletion) was not detected. DNA sequencing further demonstrated the inclusive expression of exon 18 in the Nav1.5 cDNA (data of sequencing not shown). These results indicate that Nav1.5a may not be expressed within the frontal lobe cortex of the human brain, or it is expressed at a very low level.

Expression of Nav1.5b in the human brain cortex. Primer P4 that targeted the full lengths of exons 17 and 18 of Nav1.5 cDNA was used to detect the expression of Nav1.5b. The expected fragment size of $1,123 \mathrm{bp}$ was observed following electrophoresis (Fig. 4). The fragment with alternative splicing of exon 17 and 18 (600-bp deletion) was not observed on the agarose gel. This result suggests that Nav1.5b may not be expressed within the frontal lobe cortex of the human brain, or it is expressed at a very low level.

Expression of alternatively spliced variant Q1077 (Nav1.5c) and Q1077del (wild Nav1.5) in the human brain cortex. Primers P3, that targeted partial exon 17 and full exon 18, and P5, that targeted the conjunction region of exons 17 and 18 , were used to investigate the expression of Nav1.5c and wild Nav1.5 (Nav1.5) in the frontal lobe cortex of the human brain, respectively. Direct DNA sequencing was performed on the PCR products generated by primer P3. As demonstrated in Fig. 5, the results indicated that a single sequence presented in the coding region of exons 17 and 19, whereas a dual sequence presented in the exon 18 coding region (Fig. 5A). Sequence analysis demonstrated that two CAG repeats in the conjunction region of exons 17 and 18 led to the alternative splicing of a glutamine $(\mathrm{Q})$ at position 1,077, indicating that Nav1.5c and wild Nav1.5 were expressed in the human brain
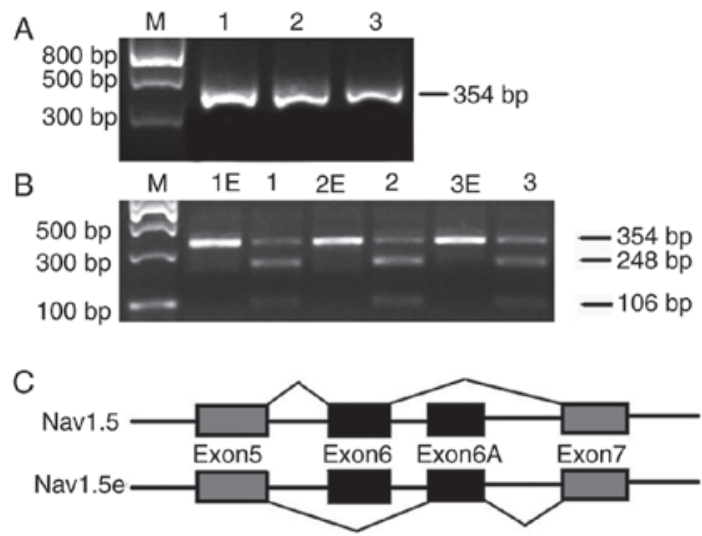

Figure 2. Alternative splicing of exon 6 and $6 \mathrm{~A}$ in the SCN5A gene. (A) Agarose gel depicting the electrophoresis results following polymerase chain reaction with primer P2. A single, clear band with the expected size of 354 bp was observed. Lanes 1-3, samples of human brain cortex from the frontal lobe. (B) Electrophoresis results following digestion by restriction enzyme SacI. Products with restriction enzyme $S a c I$ generated three bands on the agarose gel, with the expected sizes of 354, 248 and $106 \mathrm{bp}$, respectively, indicating that fragments including exon $6 \mathrm{~A}$ were digested into another two fragments, while those containing exon 6 were preserved. Lanes 1E-3E, PCR products with restriction enzyme SacI; lanes 1-3, PCR products without restriction enzyme $\operatorname{SacI}$. (C) Schematic for the alternative splicing of exons 6 and $6 \mathrm{~A}$ of the SCN5A gene. Constitutive exons are presented in gray boxes, alternatively spliced sequences are presented in solid boxes and introns are presented as solid lines. The two different pathways are presented as poly lines. M, marker.

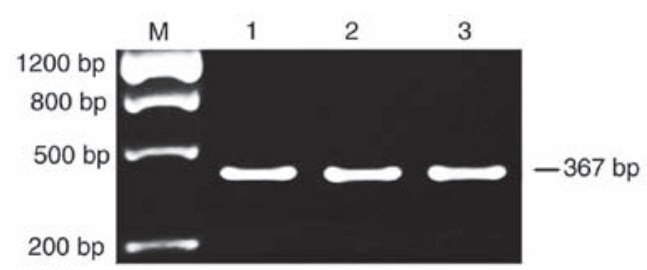

Figure 3. Polymerase chain reaction results with primer P3 targeting Nav1.5a. Electrophoresis results demonstrated that only one band with the expected size of 367 bp was observed on the agarose gel. M, marker; lanes 1-3, samples of human brain cortex from the frontal lobe.

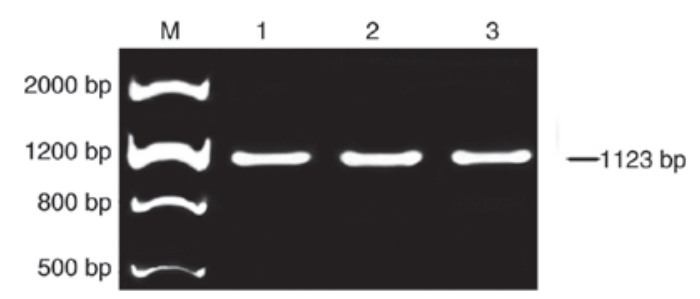

Figure 4. Polymerase chain reaction results with primer P4 targeting Nav1.5b. Electrophoresis results demonstrated that only one band with the expected size 1,123 bp was observed on the agarose gel. M, marker; lanes 1-3, samples of human brain cortex from the frontal lobe.

cortex (Fig. 5C). In order to determine the expression quantifications of the two Nav1.5 variants, primer P4 was used. It targeted (harboring) the conjunction region of exons 17 and 18; however, it generated shorter products than expected (Fig. 5B). Electrophoresis revealed the presence of transcripts with 132 and $135 \mathrm{bp}$. Autoradiography was used for signal quantification of the two alternatively spliced transcripts. The proportion 


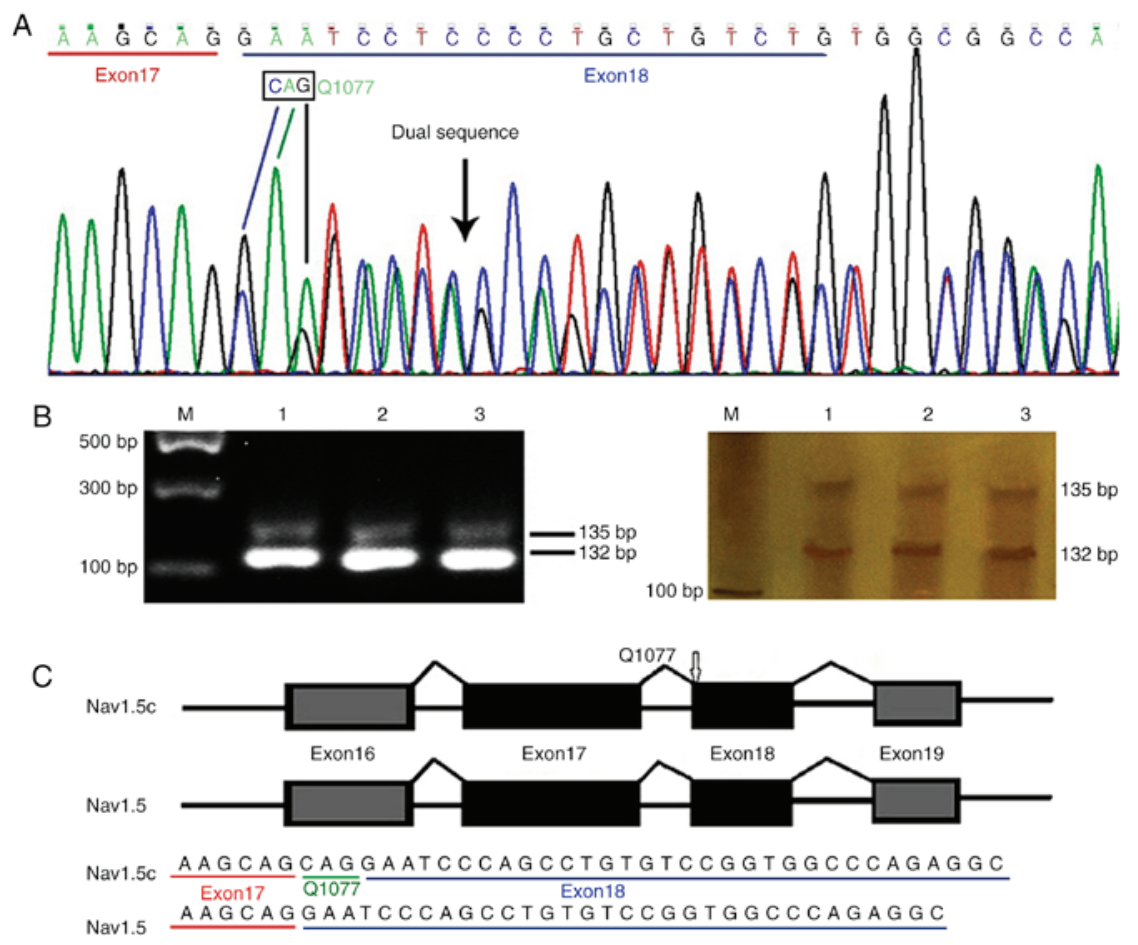

Figure 5. Alternative splicing of Q1077 (Nav1.5c). (A) DNA sequencing results for polymerase chain reaction products using primer P3. A single sequence presented in the coding region of exons 17 and 19, and a dual sequence presented in the coding region of exon 18, as indicated by the arrow. This indicated the coexistence of Nav1.5c and wild Nav1.5 in the human brain tissue. (B) Agarose and denaturing gel electrophoresis results with primer P5 demonstrated the presence of transcripts with 132 and $135 \mathrm{bp}$. (C) Schematic for the alternative splicing of Nav1.5c. Q1077 was inserted in the starting site of exon 18. Constitutive exons are indicated by gray boxes, alternatively spliced sequences are indicated by solid boxes and introns are indicated as solid lines. The two different pathways are presented as poly lines. M, marker; lanes 1-3, samples of human brain cortex from the frontal lobe.

of alternatively spliced variants containing Q1077 was $~ 16.6 \%$ $(\mathrm{n}=5)$ and the Q1077del transcript was $\sim 83.4 \%(\mathrm{n}=5)$, the expression ratio of Nav1.5:Nav1.5c was $\sim 5: 1$ (data of expression ratio not shown).

Expression of Nav1.5d in the human brain cortex. Primer P6 targeting the full length of exon 17 was used to detect the expression of Nav1.5d, the variant generated by partial alternative splicing of exon 17 (with an intermediate 120-bp deletion). PCR products with the expected fragment size of 373 bp were observed on $2 \%$ agarose gel (Fig. 6A); however, the expected fragments of $253 \mathrm{bp}$ were not observed. Direct DNA sequencing further confirmed the inclusive expression of the full-length exon 17 (Fig. 6B). This result was the same as that obtained using primer P3, indicating no or very low expression of Nav1.5d in the frontal lobe cortex of the human brain.

Expression and distribution of total Nav1.5 protein in the neurons and glial cells of gray and white matter within the human brain cortex. Tissue staining and immunohistochemistry were used to investigate the expression and distribution of total Nav1.5 protein in the neurons and glial cells of the frontal lobe cortex of the human brain (Fig. 7). The results revealed that Nav1.5 immunoreactivity was predominantly located within the neuronal cell bodies and processes, including the axons and dendrites, whereas little or no immunoreactivity was detected in the glial components. Pyramid cells in layer V of the frontal lobe cortex of the human brain revealed slightly stronger immunoreactivity to Nav1.5 antibody compared with neurons in the other layers (Fig. 7). The results reveal the expression and distribution of Nav1.5 at the protein level in the frontal lobe cortex of the human brain.

\section{Discussion}

The VGSC isoform Nav1.5, encoded by the SCN5A gene, is the predominant sodium channel in the heart (28). It was previously referred to as the cardiac sodium channel as it is essential for action potential initiation in atrial and ventricular cardiomyocytes $(25,28)$. In 1991, Yarowsky et al (10) first detected Nav1.5 mRNA in the brain cortex of neonatal and adult rats. Throughout the following decade, several studies demonstrated the expression of Nav1.5 mRNA, protein or its TTX-resistant sodium current within mammalian brains (11-19,22). The Nav1.5 sodium channel expressed in the brain was considered to be identical to the one expressed in the adult heart (20). However, when full length Nav1.5 cDNA were cloned from the human neuroblastoma cell line NB-1 and the brain cortex, neonatal Nav1.5 was detected and confirmed as a novel variant of Nav1.5 expressed in nervous tissue, which was different in structure and function to the wild Nav1.5 located in adult human hearts $(20,23,24)$. Previous studies have demonstrated that Nav1.5 may be associated with certain neurological diseases. A study by Black et al (29) indicated a central, robust upregulation of the sodium channel Nav1.5 in reactive astrocytes at the borders of and within active and chronic multiple sclerosis lesions. Two previous studies detected a SCN5A mutation in patients with epilepsy $(30,31)$. The function of Nav1.5 within the normal human brain and its potential association with neurological diseases is being increasingly studied. 

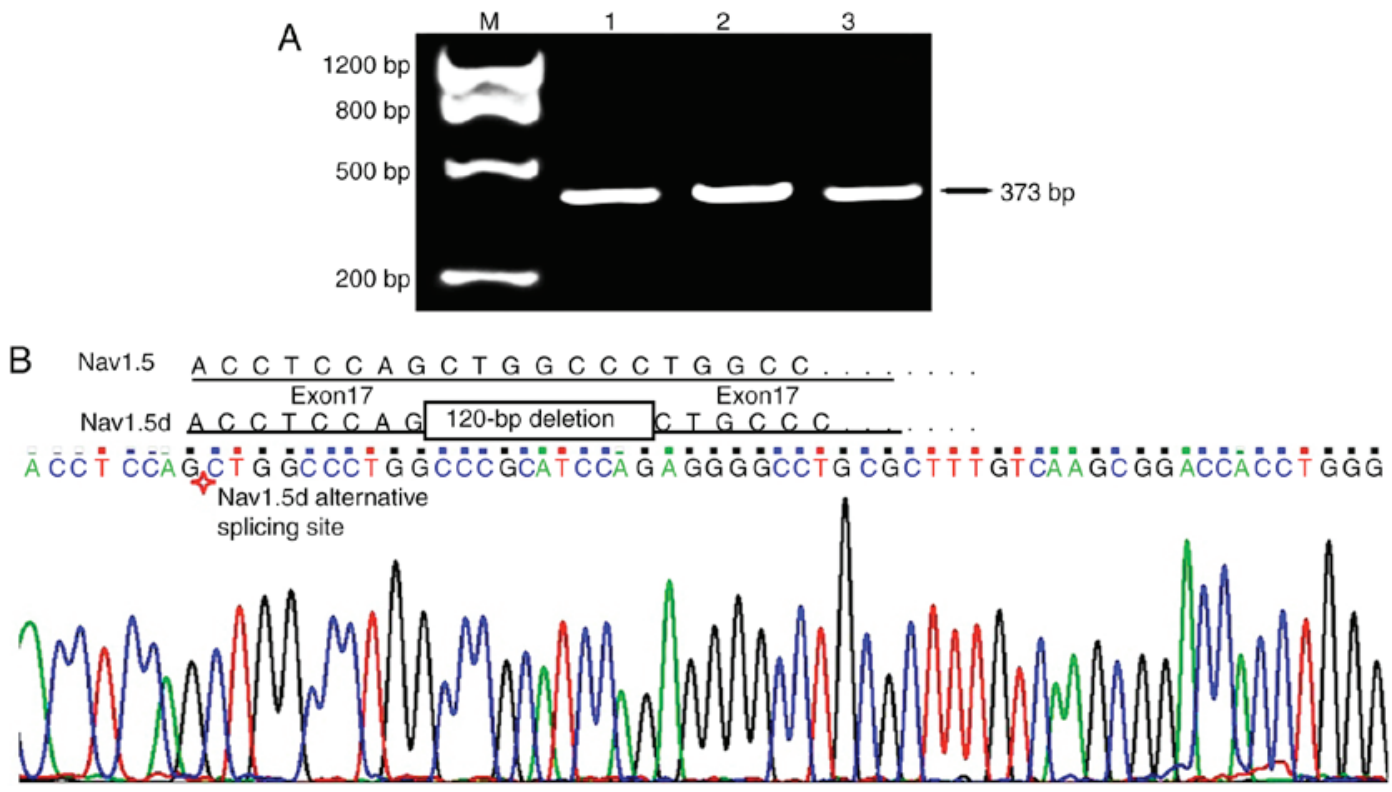

Figure 6. PCR and DNA sequencing results with primer P6 targeting Nav1.5d. (A) Electrophoresis following PCR with P6 demonstrated one band with the expected size of $373 \mathrm{bp}$. (B) Direct DNA sequencing of the PCR products confirmed the inclusive expression of the full-length exon 17. PCR, polymerase chain reaction; $\mathrm{M}$, marker; lanes 1-3, samples of human brain cortex from the frontal lobe.

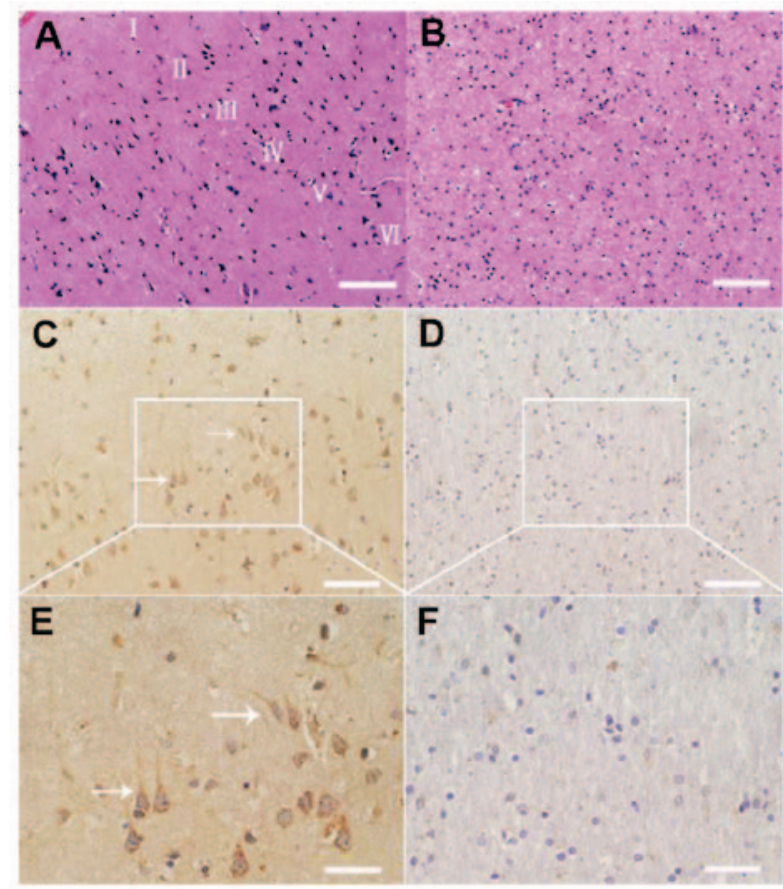

Figure 7. Immunohistochemical staining of the Nav1.5 protein in human brain tissue. (A) H\&E staining of the gray matter of samples of human brain cortex. (B) H\&E staining of the white matter of samples of human brain cortex. (C) Nav1.5 staining (dark brown) in the cortical layer. (D) Nav1.5 staining in the white matter. Scale bar, $100 \mu \mathrm{m}$. (E) Magnified image of Nav1.5 staining in the cortical layer. (F) Magnified image of Nav1.5 staining in the white matter. I-VI represents the six layers of brain cortex. Scale bar, $50 \mu \mathrm{m}$. As indicated by the arrows, Nav1.5 immunoreactivity was predominantly in neuronal processes and cell bodies, including axons and dendrites, whereas little or no immunoreactivity was detected in the glial components. H\&E, hematoxylin and eosin; I-VI, six layers of the brain cortex.

Compared with wild Nav1.5, Nav1.5a is characterized by a deletion of exon 18 (126 bp) of the SCN5A gene, which encodes 53 amino acid residues in the intracellular loop between domains II and III of the Nav1.5 channel (32). This splice variant was the first reported splice variant of the cardiac sodium channel Nav1.5, which was identified in a rat brain, heart, and hippocampal progenitor stem cells and mouse cardiac cells $(18,33)$. Subsequently, Nav1.5a expression was identified in neonatal and adult dorsal root ganglia (DRG) $(32,34)$, human neuroblastoma cell line NB-1 (20) and rat striatal progenitor cell line ST14A (35). Although Nav1.5a has been previously revealed as being expressed in several tissue types within the nervous system, including the rat brain (36), it was not detected within the human brain cortex in the present study. This result was consistent with the results of our previous study (24). Previous studies have theorized that the removal of the exon 18 sequence from the Nav1.5 transcript is specific only to small rodents; higher mammals retained this exon, suggesting an associated species-specific function $(25,37)$. Therefore, it is important to specify whether alternative splicing of exon 18 affects the kinetics of the Nav1.5 channel. Our previous study suggested that although two alternatively spliced variants, designated hNbR1 and hNbR1-2 (wild Nav1.5 and Nav1.5a cloned from human neuroblastoma cell line NB-1), demonstrated similar mRNA expression levels and sodium current, they were slightly different in the kinetics of steady-state inactivation and activation (20). Similar results were revealed by Kerr et al (32) who investigated Nav1.5a in adult mouse dorsal root ganglia. The above results of previous studies indicated that alternative splicing of exon 18 affects the electrophysiological activity of Nav1.5 channel.

Nav1.5b is characterized by alternative splicing of exons 17 and 18 encoding 200 amino acid residues in S6 of domain II, and the intracellular loop between domain II and III of the Nav1.5 channel (28). It was first detected as being expressed in very low levels in the hearts of mice (33). However, this variant was not identified in the hearts of other species, including rats, pigs and humans (37). Nav1.5b was not detected in the frontal lobe cortex of the human brain in the present study. Notably, 
previous electrophysiological recordings have revealed that Nav1.5b was a nonfunctional splice variant (25). Alternative splicing of exon 18 may generate a functional variant of Nav1.5a, therefore the existence of a partial or full exon 17 was essential to maintain the function of the Nav1.5 channel (25). It may be hypothesized that the differential expression of Nav1.5b in various tissue types may be associated with the tissue-specific expression patterns of sodium channels.

Nav1.5c is characterized by an additional CAG trinucleotide (encoding an additional glutamine at position 1077) at the starting site of exon 18, and it is the most abundant Nav1.5 splice variant detected in the heart (38). A previous study investigated the expression of Nav1.5c in the heart of humans (38). The results demonstrated that the Nav1.5:Nav1.5c transcript ratio was $\sim 2: 1$ and remained constant in different regions of the human heart (38); however, the expression level of Nav1.5c was demonstrated to be lower in mouse heart and thigh muscle, and trigeminal and dorsal root ganglia $(32,34)$. This suggested a distinct expression pattern of Nav1.5c in different species and tissue types. Our previous studies also detected the expression of Nav1.5c in the human brain cortex and neuroblastoma cell line NB-1 (20,24). However, the associated Nav1.5c transcript level in those tissues was not detected simultaneously, as an indirect DNA sequencing method was used to analyze the PCR products (24), which had limitations. The method used in the present study was improved compared with previous studies, the PCR products of the present study were purified and sequenced directly, meaning the dual cDNA sequences in the products were detected. DNA sequence analysis revealed that wild Nav1.5 and Nav1.5c were expressed in the frontal lobe cortex of the human brain. Electrophoresis on agarose gel and denaturing gel electrophoresis on a polyacrylamide/urea gel were used independently to determine the expression quantifications of the two splice variants. The expression ratio of the two variants in the human brain cortex was $~ 5: 1$, which was similar to that identified in the adult mouse DRG, but different from that identified in the adult human heart $(32,38)$. These results indicate that the tissue diversity may not affect the specific expression pattern of Nav1.5 channels. Previous studies demonstrated that the electrophysiological properties of wild Nav1.5 and Nav1.5c variants were indistinguishable when explored in the same wild-type background Nav1.5 cDNA $(25,38-40)$. However, as demonstrated by previous studies on SCN5A channelopathies, including SCN5A mutations in Long QT syndrome or Brugada syndrome, the channel kinetics of Nav1.5 are altered significantly when expressing the mutations in wild Nav1.5 compared with that in Nav1.5c background (25,39-41), indicating a different role of the respective splicing variants of Nav1.5 in SCN5A channelopathies.

Nav1.5d is characterized by a deletion of a 120-bp fragment in the intermediate region of exon 17 of gene SCN5A. A previous study identified this when exploring the expression of Nav1.5a and Nav1.5b in the human heart (42). The electrophysiological analysis revealed that significant changes occurred in channel kinetics, including the depolarized shift of steady-state activation and inactivation, and the reduction of whole cell current density when Nav1.5d was present (42). This result demonstrated that even partial alternative splicing of exon 17 may alter the function of Nav1.5 channels. To the best of our knowledge, this splice variant has not been detected in the hearts of mice, rats, pigs or dogs $(37,42)$. In the present study Nav1.5d was not detected in the human brain cortex, suggesting that Nav1.5d may not be expressed in the frontal lobe cortex of the human brain, or it is only present at very low levels. This result further suggested a tissue-specific expression pattern of sodium channel isoforms.

Nav1.5e (neonatal Nav1.5) is encoded by exon 6A of the SCN5A gene, while wild Nav1.5 (adult Nav1.5) is encoded by exon 6 of the SCN5A gene (20,21,23-25). Gene sequence analysis has revealed that exon 6 and $6 \mathrm{~A}$ of the human SCN5A gene has $92 \mathrm{bp}$, which encodes 30 amino acid residues, 7 of which were different between them (20,21,23-25). Nav1.5e splice variant has been previously observed in various cells and tissues, including the human neuroblastoma cell line NB-1 (20), human brain astrocytoma (43), breast cancer tissue $(44,45)$, human and mouse brain tissue $(21,23,24,36)$, neonatal mouse heart and several other types of cancer tissue $(21,46)$. The present study revealed that adult and neonatal Nav1.5 are expressed within the frontal lobe cortex of the human brain. As aforementioned, a major reason for not detecting the expression of adult Nav1.5 in the human brain cortex in previous studies was the use of an indirect DNA sequencing method to test the PCR products. Therefore, certain fragments in the PCR products were missed following subcloning them into a PGEM-T-easy vector, as it was impossible to test every colony. In the present study, the PCR products were sequenced directly following purification and the results suggested that adult and neonatal Nav1.5 are expressed in the human brain cortex. Notably, the expression quantification of the two splice variants of Nav1.5 was different; the expression ratio of neonatal Nav1.5 vs. wild Nav1.5 was 5:1. This result indicates that Nav1.5e is the major Nav1.5 channel in the frontal lobe cortex of the human brain. The total expression level of Nav1.5 in the human brain cortex is notably lower than that in the heart (23). According to the results of the present study, adult Nav1.5 accounts for just $1 / 6$ of the total Nav1.5, explaining why no signal appeared in the agarose gel when specific PCR primers were used in our previous study (23). To the best of our knowledge, the present study is the first to demonstrate the expression of neonatal and adult Nav1.5 isoforms in the human brain cortex, therefore indicating the abundant expression of VGSC isoforms in the CNS.

Confirmation of the expression of neonatal and adult Nav1.5 isoforms in the human brain is critical, as these two splice variants exhibit distinct electrophysiological properties. Onkal et al (26) systematically investigated the channel kinetics of neonatal and adult Nav1.5 and demonstrated that the neonatal channel exhibited a depolarized threshold of activation and voltage at which the current peaked, slower kinetics of activation and inactivation, $50 \%$ greater transient charge influx, a stronger voltage dependence of time to peak and a slower recovery from inactivation compared to adult Nav1.5. These results combined with the results of the present study suggest that the TTX-resistant sodium current in human brain neurons is generated by neonatal and adult Nav1.5 channels. Further studies are required to demonstrate the specific contributions of each Nav1.5 splice variant to the total Nav1.5 sodium channel function in neurons.

It has been previously hypothesized that Nav1.5 may have an impact on seizure activity as it has been widely identified 
in the limbic regions of the brain, which have a close association with epilepsy $(16,23)$. Additionally, mutations in the human heart sodium channel SCN5A gene have been identified to cause cardiac abnormalities, including congenital Long QT 3 syndrome and idiopathic ventricular fibrillation (28). Furthermore, myocardial and neural cells exhibit similarities in electrophysiological activities, such as the spontaneous generation of action potential (4). This hypothesis has been supported by several studies, including a study by Aurlien et al (30) that first reported a novel missense mutation (R523C) in the SCN5A gene in a patient with idiopathic epilepsy who suffered mortality. Parisi et al (31) first reported a coexistence of epilepsy and Brugada syndrome in a family with an SCN5A mutation. These previous studies presented a novel view of Nav1.5 expression, however, further studies are required to investigate the potential causative association between SCN5A mutation and epilepsy.

In conclusion, the present study demonstrated the expression of wild Nav1.5, Nav1.5c, and Nav1.5e in the human brain cortex, indicating that the TTX-resistant sodium current was a compound product of different Nav1.5 variants. The present study also demonstrated a more abundant and complicated expression of Nav1.5 in the human brain than previously reported, and provided insights into the functional significance and complexity of Nav1.5 sodium channels in neurons. Further studies are required to explore the specific contributions of Nav1.5 and its splice variants to the total sodium current and excitability of neurons.

\section{Acknowledgements}

The present study was supported by the National Natural Science Foundation of China (grant no. 31100770) and the Liaoning Provincial Natural Science Foundation of China (grant no. 2014021097).

\section{References}

1. Eijkelkamp N, Linley JE, Baker MD, Minett MS, Cregg R, Werdehausen R, Rugiero F and Wood JN: Neurological perspectives on voltage-gated sodium channels. Brain 135: 2585-2612, 2012.

2. Hu W, Tian C, Li T, Yang M, Hou H and Shu Y: Distinct contributions of $\mathrm{Na}(\mathrm{v}) 1.6$ and $\mathrm{Na}(\mathrm{v}) 1.2$ in action potential initiation and backpropagation. Nat Neurosci 12: 996-1002, 2009.

3. Kole MH, Ilschner SU, Kampa BM, Williams SR, Ruben PC and Stuart GJ: Action potential generation requires a high sodium channel density in the axon initial segment. Nat Neurosci 11: 178-186, 2008.

4. Catterall WA: From ionic currents to molecular mechanisms: The structure and function of voltage-gated sodium channels. Neuron 26: 13-25, 2000.

5. Yu FH, Yarov-Yarovoy V, Gutman GA and Catterall WA: Overview of molecular relationships in the voltage-gated ion channel superfamily. Pharmacol Rev 57: 387-395, 2005.

6. Catterall WA: Voltage-gated sodium channels at 60: Structure, function and pathophysiology. J Physiol 590: 2577-2589, 2012.

7. Cummins TR, Aglieco F, Renganathan M, Herzog RI, Dib-Hajj SD and Waxman SG: Nav1.3 sodium channels: Rapid repriming and slow closed-state inactivation display quantitative differences after expression in a mammalian cell line and in spinal sensory neurons. J Neurosci 21: 5952-5961, 2001.

8. Noda M, Ikeda T, Kayano T, Suzuki H, Takeshima H, Kurasaki M, Takahashi $\mathrm{H}$ and Numa S: Existence of distinct sodium channel messenger RNAs in rat brain. Nature 320: 188-192, 1986.

9. Schaller KL, Krzemien DM, Yarowsky PJ, Krueger BK and Caldwell JH: A novel, abundant sodium channel expressed in neurons and glia. J Neurosci 15: 3231-3242, 1995.
10. Yarowsky PJ, Krueger BK, Olson CE, Clevinger EC and Koos RD: Brain and heart sodium channel subtype mRNA expression in rat cerebral cortex. Proc Natl Acad Sci USA 88: 9453-9457, 1991

11. White JA, Alonso A and Kay AR: A heart-like $\mathrm{Na}^{+}$current in the medial entorhinal cortex. Neuron 11: 1037-1047, 1993.

12. Hoehn K, Watson TW and MacVicar BA: A novel tetrodotoxin-insensitive, slow sodium current in striatal and hippocampal neurons. Neuron 10: 543-552, 1993.

13. Zeng D, Kyle JW, Martin RL, Ambler KS and Hanck DA: Cardiac sodium channels expressed in a peripheral neurotumor-derived cell line, RT4-B8. Am J Physiol 270: C1522-C1531, 1996.

14. Deisz RA: A tetrodotoxin-insensitive [corrected] sodium current initiates burst firing of neocortical neurons. Neuroscience 70: 341-351, 1996.

15. Gu XQ, Dib-Hajj S, Rizzo MA and Waxman SG: TTX-sensitive and -resistant $\mathrm{Na}^{+}$currents, and mRNA for the TTX-resistant rH1 channel, are expressed in B104 neuroblastoma cells. J Neurophysiol 77: 236-246, 1997.

16. Hartmann HA, Colom LV, Sutherland ML and Noebels JL: Selective localization of cardiac SCN5A sodium channels in limbic regions of rat brain. Nat Neurosci 2: 593-595, 1999.

17. Donahue LM, Coates PW, Lee VH, Ippensen DC, Arze SE and Poduslo SE: The cardiac sodium channel mRNA is expressed in the developing and adult rat and human brain. Brain Res 887: $335-343,2000$

18. Gersdorff Korsgaard MP, Christophersen P, Ahring PK and Olesen SP: Identification of a novel voltage-gated $\mathrm{Na}^{+}$channel $\mathrm{rNa}(\mathrm{v}) 1.5 \mathrm{a}$ in the rat hippocampal progenitor stem cell line HiB5. Pflugers Arch 443: 18-30, 2001.

19. Wu L, Nishiyama K, Hollyfield JG and Wang Q: Localization of Nav1.5 sodium channel protein in the mouse brain. Neuroreport 13: 2547-2551, 2002.

20. Ou SW, Kameyama A, Hao LY, Horiuchi M, Minobe E, Wang WY, Makita N and Kameyama M: Tetrodotoxin-resistant $\mathrm{Na}^{+}$channels in human neuroblastoma cells are encoded by new variants of Nav1.5/SCN5A. Eur J Neurosci 22: 793-801, 2005.

21. Chioni AM, Fraser SP, Pani F, Foran P, Wilkin GP, Diss JK and Djamgoz MB: A novel polyclonal antibody specific for the $\mathrm{Na}(\mathrm{v}) 1.5$ voltage-gated $\mathrm{Na}(+)$ channel 'neonatal' splice form. J Neurosci Methods 147: 88-98, 2005.

22. Frenz CT, Hansen A, Dupuis ND, Shultz N, Levinson SR, Finger TE and Dionne VE: NaV1.5 sodium channel window currents contribute to spontaneous firing in olfactory sensory neurons. J Neurophysiol 112: 1091-104, 2014.

23. Wang J, Ou SW, Wang YJ, Zong ZH, Lin L, Kameyama M and Kameyama A: New variants of Nav1.5/SCN5A encode $\mathrm{Na}^{+}$ channels in the brain. J Neurogenet 22: 57-75, 2008.

24. Wang J, Ou SW, Wang YJ, Kameyama M, Kameyama A and Zong ZH: Analysis of four novel variants of Nav1.5/SCN5A cloned from the brain. Neurosci Res 64: 339-347, 2009.

25. Schroeter A, Walzik S, Blechschmidt S, Haufe V, Benndorf K and Zimmer T: Structure and function of splice variants of the cardiac voltage-gated sodium channel $\mathrm{Na}(\mathrm{v}) 1.5$. J Mol Cell Cardiol 49: 16-24, 2010.

26. Onkal R, Mattis JH, Fraser SP, Diss JK, Shao D, Okuse K and Djamgoz MB: Alternative splicing of Nav1.5: An electrophysiological comparison of 'neonatal' and 'adult' isoforms and critical involvement of a lysine residue. J Cell Physiol 216: 716-726, 2008.

27. Walzik S, Schroeter A, Benndorf K and Zimmer T: Alternative splicing of the cardiac sodium channel creates multiple variants of mutant T1620K channels. PLoS One 6: e19188, 2011.

28. Rook MB, Evers MM, Vos MA and Bierhuizen MF: Biology of cardiac sodium channel Nav1.5 expression. Cardiovasc Res 93: 12-23, 2012.

29. Black JA, Newcombe J and Waxman SG: Astrocytes within multiple sclerosis lesions upregulate sodium channel Nav1.5. Brain 133: 835-846, 2010.

30. Aurlien D, Leren TP, Tauboll E and Gjerstad L: New SCN5A mutation in a SUDEP victim with idiopathic epilepsy. Seizure 18: 158-160, 2009.

31. Parisi P, Oliva A, Coll Vidal M, Partemi S, Campuzano O, Iglesias A, Pisani D, Pascali VL, Paolino MC, Villa MP, et al: Coexistence of epilepsy and Brugada syndrome in a family with SCN5A mutation. Epilepsy Res 105: 415-418, 2013.

32. Kerr NC, Gao Z, Holmes FE, Hobson SA, Hancox JC, Wynick D and James AF: The sodium channel Nav1.5a is the predominant isoform expressed in adult mouse dorsal root ganglia and exhibits distinct inactivation properties from the full-length Nav1.5 channel. Mol Cell Neurosci 35: 283-291, 2007. 
33. Zimmer T, Bollensdorff C, Haufe V, Birch-Hirschfeld E and Benndorf $\mathrm{K}$ : Mouse heart $\mathrm{Na}^{+}$channels: Primary structure and function of two isoforms and alternatively spliced variants. Am J Physiol Heart Circ Physiol 282: H1007-H1017, 2002.

34. Kerr NC, Holmes FE and Wynick D: Novel isoforms of the sodium channels Nav1.8 and Nav1.5 are produced by a conserved mechanism in mouse and rat. J Biol Chem 279: 24826-24833, 2004.

35. Wasner U, Geist B, Battefeld A, Bauer P, Müller J, Rolfs A and Strauss U: Specific properties of sodium currents in multipotent striatal progenitor cells. Eur J Neurosci 28: 1068-1079, 2008.

36. Ren CT, Li DM, Ou SW, Wang YJ, Lin Y, Zong ZH, Kameyama M and Kameyama A: Cloning and expression of the two new variants of Nav1.5/SCN5A in rat brain. Mol Cell Biochem 365: 139-148, 2012.

37. Blechschmidt S, Haufe V, Benndorf $\mathrm{K}$ and Zimmer T: Voltage-gated $\mathrm{Na}^{+}$channel transcript patterns in the mammalian heart are species-dependent. Prog Biophys Mol Biol 98: 309-318, 2008.

38. Makielski JC, Ye B, Valdivia CR, Pagel MD, Pu J, Tester DJ and Ackerman MJ: A ubiquitous splice variant and a common polymorphism affect heterologous expression of recombinant human SCN5A heart sodium channels. Circ Res 93: 821-828, 2003

39. Tan BH, Valdivia CR, Rok BA, Ye B, Ruwaldt KM, Tester DJ, Ackerman MJ and Makielski JC: Common human SCN5A polymorphisms have altered electrophysiology when expressed in Q1077 splice variants. Heart Rhythm 2: 741-747, 2005.

40. Tan BH, Valdivia CR, Song C and Makielski JC: Partial expression defect for the SCN5A missense mutation G1406R depends on splice variant background Q1077 and rescue by mexiletine. Am J Physiol Heart Circ Physiol 291: H1822-H1828, 2006.
41. Wang DW,DesaiRR,CrottiL,ArnestadM,InsoliaR,PedrazziniM, Ferrandi C, Vege A, Rognum T, Schwartz PJ and George AL Jr: Cardiac sodium channel dysfunction in sudden infant death syndrome. Circulation 115: 368-376, 2007.

42. Camacho JA, Hensellek S, Rougier JS, Blechschmidt S, Abriel H, Benndorf K and Zimmer T: Modulation of Nav1.5 channel function by an alternatively spliced sequence in the DII/DIII linker region. J Biol Chem 281: 9498-9506, 2006.

43. Xing D, Wang J, Ou S, Wang Y, Qiu B, Ding D, Guo F and Gao Q: Expression of neonatal Nav1.5 in human brain astrocytoma and its effect on proliferation, invasion and apoptosis of astrocytoma cells. Oncol Rep 31: 2692-2700, 2014.

44. Brackenbury WJ, Chioni AM, Diss JK and Djamgoz MB: The neonatal splice variant of Nav1.5 potentiates in vitro invasive behaviour of MDA-MB-231 human breast cancer cells. Breast Cancer Res Treat 101: 149-160, 2007.

45. Fraser SP, Diss JK, Chioni AM, Mycielska ME, Pan H, Yamaci RF, Pani F, Siwy Z, Krasowska M, Grzywna Z, et al: Voltage-gated sodium channel expression and potentiation of human breast cancer metastasis. Clin Cancer Res 11: 5381-5389, 2005.

46. House CD, Vaske CJ, Schwartz AM, Obias V, Frank B Luu T, Sarvazyan N, Irby R, Strausberg RL, Hales TG, et al: Voltage-gated $\mathrm{Na}^{+}$channel SCN5A is a key regulator of a gene transcriptional network that controls colon cancer invasion. Cancer Res 70: 6957-6967, 2010

This work is licensed under a Creative Commons Attribution-NonCommercial-NoDerivatives 4.0 International (CC BY-NC-ND 4.0) License. 